\title{
Unruptured Left Ventricular Pseudoaneurysm After Silent Myocardial Infarction with Mitral Valve Regurgitation
}

\author{
Tamer Kehibar ${ }^{1}$, Fatih KIZILYEL ${ }^{2}$, Mehmet Yilmaz ${ }^{2}$, and Bulent Ketenci ${ }^{3}$ \\ ${ }^{1}$ Prof Dr Siyami Ersek GKDCM \\ ${ }^{2}$ Dr Siyami Ersek Thoracic and Cardiovascular Training and Research Hospital \\ ${ }^{3}$ Dr. Siyami Ersek Thoracic and Cardiovascular Surgery Training and Research Hospital
}

May 31, 2021

\begin{abstract}
Acquired left ventricular pseudoaneurysm is a rare disorder that occurs after acute myocardial infarction. We present a 55 year-old male patient with a nonruptured pseudoaneurysm after acute MI presenting with severe mitral regurgitation. After resection of sac-like lesion, the defect was $5 \mathrm{~cm}$ diameter posterolaterally left ventricular. The aneursym was repaired with pericardium patch to maintain cardiac geometry that diminishes mitral regurgitation without intervention to mitral valve.
\end{abstract}

Title: Unruptured Left Ventricular Pseudoaneurysm After Silent Myocardial Infarction with Mitral Valve Regurgitation

Running Head: LVPSA case with unusual correcting mitral failure

Authors ;

1. Tamer Kehlibar, ORCID ID: 0000-0002-5274-7654, Specialist Dr.,tamerkehlibar@gmail.com, Department of Cardiovascular Surgery, Dr SiyamiErsek Thoracic and Cardiovascular Surgery Training and Research Hospital, Istanbul, Turkey

2. FatihKizilyel, ORCID ID: 0000-0001-8299-371X, Specialist Dr.,fkizilyel@gmail.com, Department of Cardiovascular Surgery, Dr SiyamiErsek Thoracic and Cardiovascular Surgery Training and Research Hospital, Istanbul, Turkey

3. Mehmet Yilmaz, ORCID ID: 0000-0002-6243-6876, Specialist Dr.,drmehmetyilmaz@gmail.com, Department of Cardiovascular Surgery, Dr SiyamiErsek Thoracic and Cardiovascular Surgery Training and Research Hospital, Istanbul, Turkey

4. BulendKetenci, ORCID ID: 0000-0002-8493-0154, Associate Professor Dr.,bulend.ketenci@saglik.gov.tr , Department of Cardiovascular Surgery, Dr SiyamiErsek Thoracic and Cardiovascular Surgery Training and Research Hospital, Istanbul, Turkey

Corresponding Author : TamerKehlibar, Specialist Dr., tamerkehlibar@gmail.comTibbiye Cd No 13, Haydarpasa, 34668 Uskudar, Istanbul, Turkey

Funding: None

Conflict of Interest: None

Unruptured Left Ventricular Pseudoaneurysm After Silent Myocardial Infarction with Mitral Valve Regurgitation

Abstract 
Acquired left ventricular pseudoaneurysm is a rare disorder that occurs after acute myocardial infarction. We present a 55 year-old male patient with a nonruptured pseudoaneurysm after acute MI presenting with severe mitral regurgitation. After resection of sac-like lesion, the defect was $5 \mathrm{~cm}$ diameter posterolaterally left ventricular. The aneursym was repaired with pericardium patch to maintain cardiac geometry that diminishes mitral regurgitation without intervention to mitral valve.

IntroductionLeft ventricular pseudoaneurysm is a rare complication of acute myocardial infarction that can result with catastrophic conditions. We present a 55 year-old male patient with a nonruptured pseudoaneurysm after acute MI presenting with severe mitral regurgitation due to the localisation of defect.

Surgical resection of the sac and repair of the ventricle was performed with bovine pericardium, also left internal mammary artery (ITA) to left anterior descending (LAD) artery bypass performed.

This article is based on previously conducted studies and does not contain any studies with human participants or animals performed by any of the authors. Informed consent has been taken by the patient and the study has been approved by the ethics committee of our hospital.Case ReportA 55 year-old male patient presented to our clinic with dyspnea and palpitation that get worse for couple of weeks. He has a history of hypertension, diabetes mellitus and chronic obstructive pulmonary disease. His blood pressure was 110/70 $\mathrm{mm} \mathrm{Hg}$, pulse rate 90 with sinus ryhtm. Transthoracic echocardiography showed $40-45 \%$ of ejection fraction, mild pericardial effusion, severe mitral regurgitation and the impairment on left ventricular segmental wall motion with a dyskinetic cavity posterolaterally. Color doppler showed passage of blood from left ventricular cavity to pericardium with and narrow opening. CT- Angiography revealed a $5 \times 5.5 \mathrm{~cm}$ sized focal sac like lesion next to posterolateral left ventricular wall with $2 \mathrm{~cm}$ neck. [Figure 1 ] Patient underwent coronary angiography, that showed $80 \%$ lesion on proximal LAD, total occlusion on circumflex artery and $70 \%$ lesion on right coronary artery $(\mathrm{RCA})$.

At surgery, a large nonruptured posterolateral pseudoaneurysm was seen. After resection of the sac, the defect was between two papillary muscle on posterior left ventricle was closed with bovine pericardium patch strengthened with teflon-felt pledgets [Figure 2 ]. Concomitant Left ITA to LAD bypass were performed. Circumflex artery was not bypass-able and RCA could not be visualisized due to adhesions. Intraaortic balloon pump was placed perioperatively. Control transesophageal echocardiography showed no outflow from the defect and mild mitral regurgitation was detected without need of mitral valve intervention and the operation was completed.

Postoperatively, the patient was taken to the intensive care unit, was extubated on the $2^{\text {nd }}$ day of his follow-up, hemodynamically stable. Safely separated from intraaortic balloon pump after the control transtorasic echocardiography reported as \%35-40 of ejection fraction, mild mitral insufficiency and posteroseptal hipokinesia. Unfourtunately he was re-intubated $5^{\text {th }}$ day due to respiratory disstress. Examinations showed up he has Covid-19 infection. Due to respiratory failure the patient deceased $8^{\text {th }}$ day postoperation.DiscussionLeft ventricular rupture is a rare mechanical complication that seen after acute myocardial infarction. Free wall rupture causes sudden death. Rarely the rupture of the ventricle is limited with adherent fibrous pericardial tissue that results as pseudoaneurysm formation.

Pseudoaneurysms are characterized by a neck narrower than the diameter of the sac which contains organized trombus and blood with no myocardium. This is in contrast to true aneurysm, which have wider neck and progresses from endocardium to pericardium respectively [1]. Acute transmural MI is the most common cause of LV pseudoaneurysm (\%55); followed by cardiac surgery (\%33), trauma (\%7) and infection (\%5)[2]. About half of the pseudoaneurysms were posterior or inferior localisation. [3] Pseudoaneurysm have an approximately 30-40\% risk of rupture and also embolism, arhytmia that can result catastrophic conditions. Two-dimensional transthoracic echocardiography and left ventriculography are the best available test for the diagnosis. Coronary angiography findings may help distinguish the source of ischemia. Also CT-Angiography and cardiac MRI provide detailed images of the pseudoaneurysm and delineates its relation to other cardiac structures. [4] Early surgical intervention with patch closure is recommended once the 
pseudoaneurysm is detected because of the high risk of rupture.

Mitral failure associated with pseudoaneurysm is caused by mainly three factors: mitral ring dilatation, restriction of posterior mital leaflet due to ventricular dilatation, loss of contraction of ventricular wall. [7] Repair of pseudoaneurysm as in this case, may support to restoration of ventricular geometry and reestablishing mitral valvar function.ConclusionsLeft ventricular wall rupture is a mortal complication occuring $4 \%$ of patients after acute MI [5]. Rarely pseudoaneurysm information can be seen with adherent pericardial tissue. Pseudoaneurysms can be symptomatic or may also have silent progress, require suspicious examination. Once it diagnosed, it should be corrected urgently because of the high risk of complication such as heart failure, embolism or sudden death. During procedure maintaining cardiac geometry is crucial for proper cardiac physiology. [6] And this case have shown that the mitral insufficiency which was thought to be related to the LV pseudoaneurysm can be corrected with repair of the pseudoaneurysm and may not require mitral intervention.

\section{References}

1. Contuzzi R, Gatto L, Patti G, Goffredo C, D’Ambrosio A, Covino E, Chello M, Di Sciascio G. Giant left ventricular pseudoaneurysm complicating an acute myocardial infarction in patient with previous cardiac surgery: a case report. J Cardiovasc Med (Hagerstown). 2009 Jan;10(1):81-4. doi: 10.2459/JCM.0b013e32831a98e6. PMID: 19145118.

2. Prifti E, Bonacchi M, Baboci A, Giunti G, Veshti A, Demiraj A, Zeka M, Rruci E, Bejko E. Surgical treatment of post-infarction left ventricular pseudoaneurysm: Case series highlighting various surgical strategies. Ann Med Surg (Lond). 2017 Mar 9;16:44-51. doi: 10.1016/j.amsu.2017.03.013. PMID: 28386394; PMCID: PMC5369265.

3. Eren E, Bozbuga N, Toker ME, Keles C, Rabus MB, Yildirim O, Guler M, Balkanay M, Isik O, Yakut C. Surgical treatment of post-infarction left ventricular pseudoaneurysm: a two-decade experience. Tex Heart Inst J. 2007;34(1):47-51. PMID: 17420793; PMCID: PMC1847932.

4. Davutoglu V, Soydinc S, Sezen Y, Aksoy M. Unruptured giant left ventricular pseudoaneurysm complicating silent myocardial infarction in a diabetic young adult: left ventricular giant pseudoaneurysm after silent myocardial infarction. Int J Cardiovasc Imaging. 2005 Apr-Jun;21(2-3):231-4. doi: 10.1007/s10554-004-2608-2. PMID: 16015433.

5. Dubey L, Timala R, Adhikari R, Sharma S, Gautam M, Gautam S. Unruptured left ventricular pseudoaneurysm following inferior wall myocardial infarction. Cardiol J. 2012;19(5):539-42. doi: 10.5603/cj.2012.0100. PMID: 23042323.

6. Demirozu ZT, Akay MH, Frazier OH. Left ventricular pseudoaneurysm arising between two papillary muscles: repair without mitral regurgitation. J Card Surg. 2010 Nov;25(6):668-70. doi: 10.1111/j.15408191.2010.01119.x. Epub 2010 Oct 11. PMID: 21039853.

7. Falcão JL, Falcão SN, Garcia MF, Arruda AL, Hueb AC, Jatene FB, Gutierrez PS, Nicolau JC, Ramires JA, Giraldez RR. Pseudoaneurisma de ventrículo esquerdo associado a insuficiência mitral grave complicando infarto agudo do miocárdio ínfero-látero-dorsal [Left ventricular pseudoaneurysm associated to severe mitral insufficiency, complicating inferolaterodorsal acute myocardial infarction]. Arq Bras Cardiol. 2005 Jun;84(6):488-91. Portuguese. doi: 10.1590/s0066-782x2005000600011. Epub 2005 Jun 28. PMID: 16007316. 

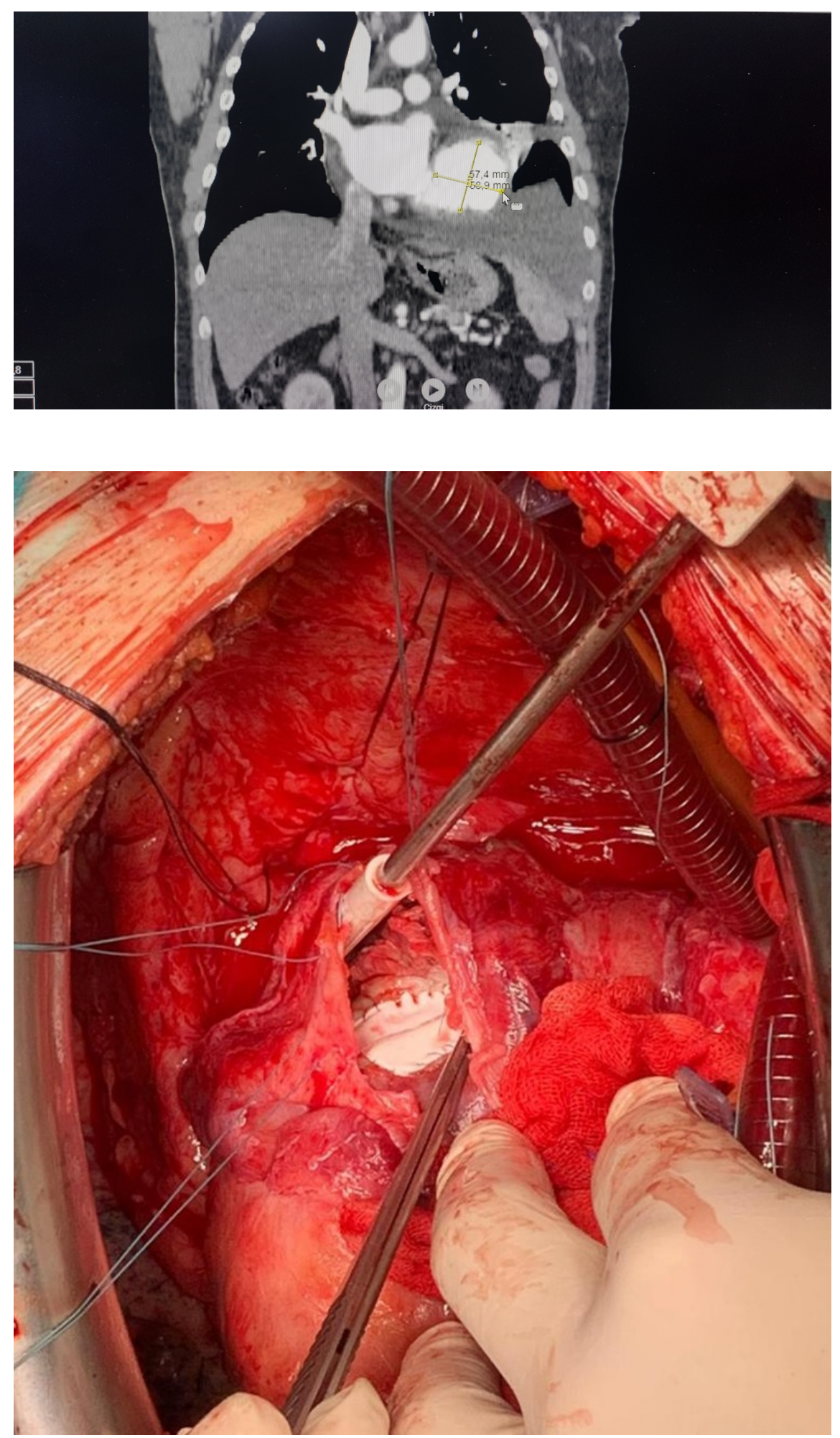